6. Surget A, Saxe M, Lema n S, et al. Drug-dependent requirement of hippocampal neurogenesis in a model of depression and of antidepressant reversal. Biol Psych 2008; 64 : 293-301.

7. Wang JW, David DJ, Monckton JE, et al. Chronic fluoxetine stimulates maturation and synaptic plasticity of adultborn hippocampal granule cells. J Neurosci $2008 ; 28$ : 1374-84.

8. Bessa JM, Ferreira D, Melo I, et al. The moodimproving actions of antidepressants do not depend on neurogenesis but are associated with neuronal remodeling. Mol Psychiatry 2009; 14 : 764-73.

9. Gourley SL, Kiraly DD, Howell JL, et al. Acute hippocampal brain-derived neurotrophic factor restores motivational and forced swin performance after corticosterone. Biol Psych 2008; 63 : 353-9.

10. Maya Vetencourt JF, Sale A, Viegi A, et al. Maffeu L. The antidepressant fluoxetine restores plasticity in the adult visual cortex. Science $2008 ; 320: 385-8$.

11. Beaulieu JM, Marion S, Rodriquiz RM, et al. A betaarrestin 2 signaling complex mediates lithium action on behavior. Cell $2008 ; 132$ : 125-36.
12. Willour VL, Chen H, Toolan J, et al. Family-based association of FKBP5 in bipolar disorder. Mol Psychiatry 2009; 14 : 261-8.

13. Dulawa SC, Holick KA, Gundersen B, Hen R. Effects of chronic fluoxetine in animal models of anxiety and depression. Neuropsychopharmacology 2004 ; 29: 1321-30

\title{
NOUVELL
}

\section{Pluripotence : une définition à géométrie variable}

Laure Coulombel

\author{
Médecine/Sciences et Inserm U935, \\ Hôpital Paul Brousse, \\ 94817 Villejuif Cedex, France. \\ laure.coulombel@inserm.fr
}

> Affirmer la pluripotence d'une cellule n'est pas chose simple; de multiples critères existent (Tableau I), qui n'ont pas la même valeur, ce qui entretient un certain «laxisme» dans la définition de cette propriété [1]. Les puristes n'accorderont ce label qu'aux cellules capables de former, à elles seules, un individu entier viable et fertile, et du bout des lèvres à celles qui contribuent «uniquement» à la formation de chimères post-natales fertiles. Critères impossibles à valider chez l'homme, et, de fait, beaucoup se refusent à qualifier de pluripotentes les cellules souches embryonnaires (CSE) humaines ou les cellules reprogrammées humaines de type iPS (induced pluripotent stem cells). Les moins exigeants se satisferont de critères phénotypiques: pour eux, une cellule qui, dans différentes conditions de culture, exprime des marqueurs de tissus dérivés des trois feuillets embryonnaires (ectoderme, endoderme, mésoderme) sera déclarée «pluripotente». Ce n'est évidemment pas suffisant compte tenu de l'expression souvent «aberrante » de beaucoup de protéines en culture; il faudrait au minimum démontrer que la cellule exprimant ces marqueurs phénotypiques d'un tissu in vitro est capable d'effectuer une fonction caractéristique de ce tissu dans un modèle in vivo; chez l'homme, on se heurte à nouveau aux limites des systèmes xénogéniques, la transplantation de cellules humaines à des souris, aussi immunodéprimées soient-elles (modèle le plus fréquemment utilisé), étant loin de refléter leur fonction physiologique chez l'humain. Cela pose avec acuité la question du choix de modèles précliniques acceptables pour l'évaluation de cellules pluripotentes humaines dans une perspective thérapeutique. Actuellement les essais les plus probants sont faits chez le singe, en situation allogénique, avec toutes les contraintes qu'impose le développement de cellules pluripotentes dans cette espèce [2].

Dans le cas des CSE (ou iPS), murines ou humaines, la formation de tératomes chez la souris immunodéficiente est un compromis acceptable pour définir la pluripotence d'une population. Mais il faut que l'analyse histologique et histochimique reconnaisse dans ces tumeurs des structures tissulaires caractéristiques (épithélium, glandes, vaisseaux, etc.) issues de la différenciation des 3 feuillets embryonnaires [3]. La capacité de créer une organisation tissulaire complexe, même anarchique, représente en effet un argument supplémentaire de pluripotence par rapport à la seule expression cellulaire d'un potentiel de différenciation dans une boîte de culture.
Une étape supplémentaire, inapplicable aux cellules humaines, exploite la capacité qu'ont ces CSE et iPS, greffées dans un blastocyste préimplantatoire, de contribuer, une fois les blastocystes « chimères » réimplantés dans des femelles pseudo-gestantes, à l'établissement d'animaux chimères viables et fertiles (Figure 1) [4-6]. Remises dans leur environnement d'origine, la masse interne des blastocystes, ces CSE reprennent en quelque sorte le cours de leur histoire naturelle là où elle s'était arrêtée lorsqu'on avait dissocié ces blastocystes pour l'établissement des lignées. Dans le cas des cellules somatiques reprogrammées (iPS), beaucoup d'eau a passé sous les ponts, depuis le stade blastocyste, et c'est une histoire complètement nouvelle qu'entament ces cellules. Dans ces chimères, la plupart des tissus, y compris les cellules germinales, contiennent des cellules différenciées issues des CSE (ou iPS); mais cette stratégie ne prouve pas que ces cellules peuvent assurer, seules, sans le support des cellules du blastocyste hôte, le développement d'animaux viables, entièrement dérivés des CSE ou iPS. Le croisement de souris chimères certes aboutira à des souriceaux entièrement dérivés des CSE ou iPS, mais dans ce cas, on analyse le potentiel des gamètes plus que celui des CSE ou iPS. 


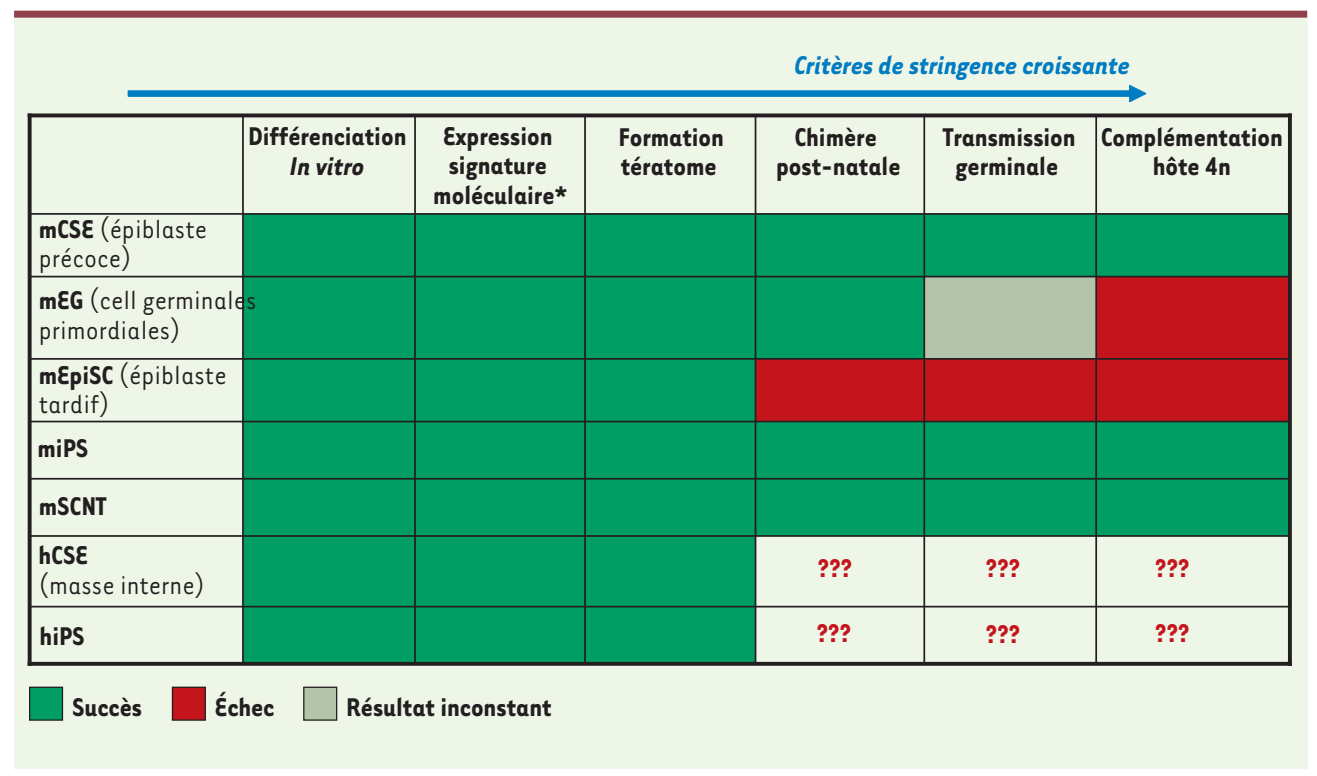

Tableau I. Différents critères utilisés pour juger de la pluripotence d'une cellule. Les critères sont classés dans un ordre hiérarchique allant du moins exigeant (à gauche) au plus rigoureux (à droite). * Signature moléculaire: au minimum expression des gènes 0ct4, sox2, nanog, ronin, tbx3. mCSE: cellule souche embryonnaire de souris ( $\mathrm{m})$; $\mathrm{m} \varepsilon G$ : lignées de cellules souches murines dérivées des embryonal gonad; mEpiSC: lignées de cellules souches murines dérivées de l'épiblaste tardif; hCSE : cellules souches embryonnaires humaines dérivées de la masse interne de blastocystes préimplantatoires; iPS: induced pluripotent stem cells (généralement dérivées de fibroblastes embryonnaires dans le cas des iPS de souris (m). mSCNT : lignées de cellules souches murines dérivées d'un transfert de noyau somatique dans des ovocytes (SCNT : somatic cell nuclear transfer).

On peut aussi obtenir le développement complet d'une souris indépendamment de la transmission germinale via des expériences dites de «complémentation d'embryons tétraploïdes », ce qui représente le gold standard des tests de pluripotence. Cela consiste à agréger les cellules à tester avec des embryons tétraploïdes (obtenus par électrofusion d'embryons au stade 2 cellules), et à réimplanter l'agrégat dans l'utérus d'une femelle gravide [7]. On peut aussi injecter les cellules à tester dans l'embryon tétraploïde après l'avoir cultivé jusqu'au stade blastocyste in vitro. A. Nagy a montré il y a vingt ans que les cellules embryonnaires tétraploïdes ne pouvaient pas (ou dans une très faible proportion et transitoirement) se développer en un embryon et contribuaient exclusivement aux annexes. Dans ces conditions, l'embryon est entièrement issu des cellules diploïdes (et seulement de 2 à 3 sur les 10-15 injectées [8]), et l'on avait envisagé dans les années 1990 que cette technique pourrait permettre d'analyser les conséquences de manipulations génétiques des CSE plus rapidement que par la stratégie des chimères transgéniques [9]. Seuls deux types de cellules embryonnaires murines étaient jusqu'à maintenant capables de complémenter des embryons tétraploïdes démontrant sans ambiguïté leur complète pluripotence: les CSE dérivées de la masse interne du blastocyste (épiblaste précoce), et les CSE obtenues par transfert de noyaux somatiques dans l'ovocyte. L'efficacité était d'environ $20 \%$ de nouveau-nés entièrement dérivés des CSE injectées dans l'embryon $4 \mathrm{n}$. $\varepsilon$ n revanche, les cellules EpiSC (epi pour epiblast) dérivées de l'épiblaste tardif (et dont les propriétés ont été suggérées proches mais pas identiques à celles de CSE humaines [10]) sont incapables de complémenter un embryon tétraploïde, confirmant probablement déjà la perte d'un certain potentiel, en accord avec le fait que l'epiblaste tardif in vivo a perdu le potentiel à générer l'ectoderme.

Dans le cas des cellules iPS, l'interrogation persistait: s'il est maintenant démontré que les iPS (murines) contribuent bien à des chimères postnatales viables et fertiles [11-14], les essais de complémentation d'embryons tétraploïdes avaient toujours échoué, les embryons ne survivant pas au-delà du stade $\varepsilon 14-\varepsilon 15$. Trois articles parus dans Nature et Cell Stem Cells, issus de deux équipes chinoises de Pékin [15] et Shanghai [16] et d'une équipe californienne [17], ont maintenant levé cet obstacle et réussi à faire naître des souriceaux dont la totalité des tissus provient de cellules iPS. Dans les trois études, les iPS provenaient de fibroblastes embryonnaires (MEF) reprogrammés «en routine» avec le «cocktail» des 4 facteurs de transcription 0ct4/Sox2/ $\mathrm{KIf4/cMyc} \mathrm{décrit} \mathrm{par} \mathrm{Yamanaka} \mathrm{[11]} \mathrm{;}$ dans deux études, les transgènes étaient insérés dans des lentivirus inductibles (sytème Tet-on), et les auteurs californiens y ont ajouté une pincée d'acide valproïque (décrit comme facilitant le processus de reprogrammation par son action au niveau épigénétique). L'efficacité de complémentation, jugée sur la naissance de souriceaux viables, est de 1 à $5 \%$ avec des variations très importantes entre les expériences: 4 souriceaux nés sur 387 embryons tétraploïdes transférés dans une étude, 27 pour 1454 embryons transférés dans la seconde ( 0 à $3,5 \%$ de succès selon les expériences), et 29 sur 1181 dans la troisième ( 0 à $13 \%$ de succès); par comparaison, 5-10\% des blastocystes 


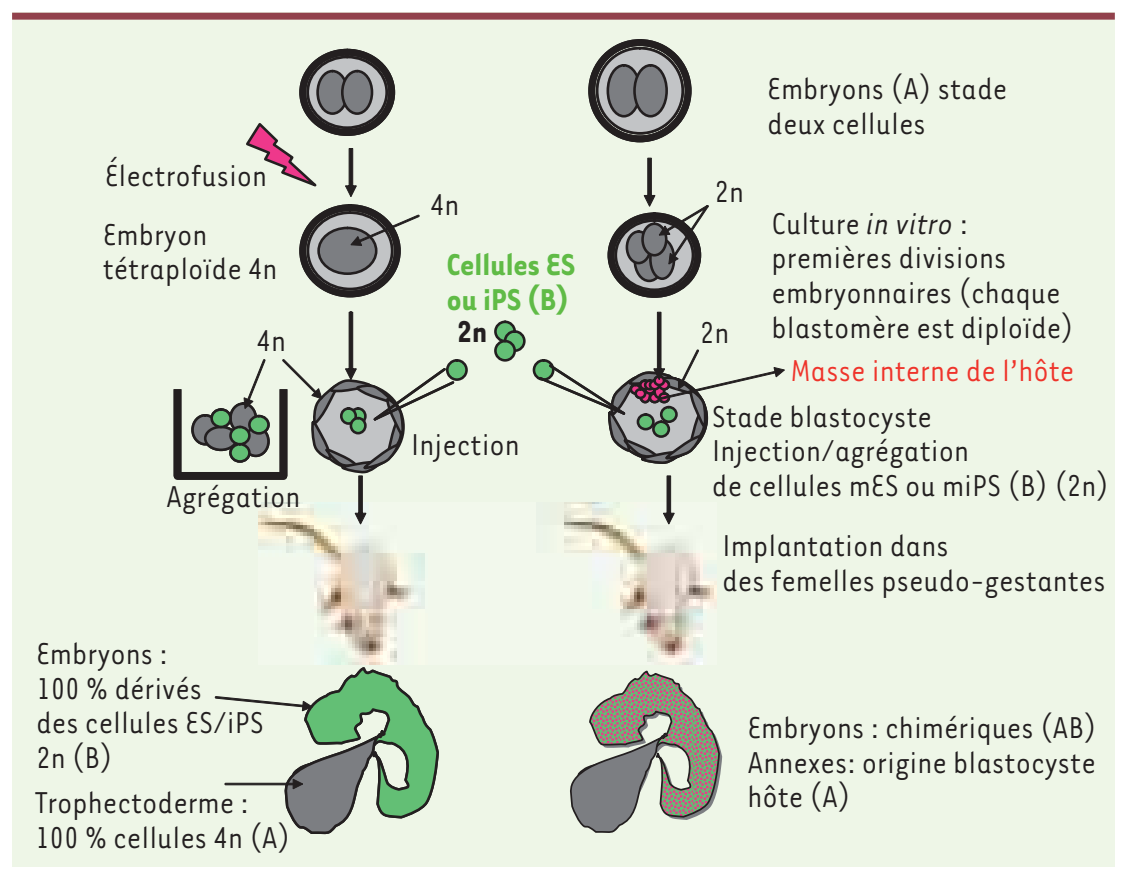

Figure 1. Les deux méthodes utilisées pour prouver la pluripotence des cellules ES ou iPS in vivo. À droite, la stratégie classique de formation de chimères : les cellules mCSE ou miPS (vert) sont injectées dans un blastocyste hôte normal, contenant sa propre masse interne (rouge). Les cellules CSE/iPS s'intègrent dans le blastocyste, qui est réimplanté dans l'utérus d'une souris pseudo-gestante. Les embryons se développent et donnent des souriceaux nouveau-nés normaux chimériques: les tissus sont dérivés des celules CSE/iPS (vert) et des cellules de la masse interne du blastocyste hôte (en rouge). En revanche, le placenta et les annexes sont dérivées uniquement du trophectoderme du blastocyste hôte (gris). À gauche, un embryon tétraploïde est créé par électrofusion d'embryons au stade 2 cellules. La fusion des blastomères entraîne la formation d'un embryon $4 \mathrm{n}$. Cette tétraploïdie compromet le développement des tissus intra-embryonnaires (la masse interne anormale $4 \mathrm{n}$ n'a pas été représentée) ; les cellules $4 \mathrm{n}$ qui se divisent sont seulement capables de se différencier en cellules extra-embryonnaires formant le placenta et les annexes ; les cellules CSE ou iPS sont injectées dans cet embryon $4 \mathrm{n}$ (ou agrégées avec lui) et celui-ci est ensuite réimplanté dans l'utérus d'une femelle pseudo-gestante. Les embryons qui se développent et qui donnent naissance à des animaux viables sont exclusivement dérivés (à $100 \%$ ) des cellules CSE ou iPS $2 n$ injectées (couleur verte des embryons). En revanche, le placenta et les annexes sont entièrement dérivées des embryons hôtes tétraploïdes (gris).

normaux dans lesquels ces mêmes iPS ont été injectées donnent naissance à des souris chimériques. Cette proportion de naissances dans les expériences de complémentation d'embryons tétraploïdes est inférieure à celle qui est observée utilisant des CSE murines.

Ces articles, suite logique d'étapes expérimentales maintenant classiques, n'ont rien de révolutionnaire, et en des temps moins «fast and furious» [18] n'auraient pas eu les honneurs de Nature, ce d'autant qu'ils ne nous donnent aucune explication sur la raison de ce succès technique attendu. Simple pugnacité, comme le suggère Rudolph Jaenisch, qui par cette même technique, n'avait pas dépassé le stade embryonnaire $\varepsilon 15$ et n'avait pas obtenu de souriceaux nouveau-né viables? Tout à fait plausible. Une autre explication nous ramène à une discussion très actuelle sur les critères de pluripotence et donc sur le caractère complet ou incomplet d'une reprogrammation, question qui est loin d'être triviale : on sait, mainte- nant que l'on a un peu (3 ans) de recul dans la connaissance de ces cellules iPS, que, selon les expériences, l'expression du cocktail magique aboutit à des lignées iPS exprimant différents degrés de pluripotence; il existe notamment une étape dite de «pré-iPS», définie initialement par l'équipe de Austin Smith, que caractérise une reprogrammation incomplète [19]. Celle-ci ne se poursuit pas spontanément, mais un coup de pouce peut être donné sous la forme d'inhibiteurs de voies de signalisation (notamment les voies ERK, GSK3 et FGFR2) décrites comme essentielles au maintien de l'autorenouvellement des cellules CSE $\rightarrow$ [20]. Les

$(\rightarrow) \mathrm{m} / \mathrm{s} 2008$ $n^{\circ} 11$, p. 934 pré-iPS sont probablement incapables de complémenter des embryons tétraploïdes, comme elles sont incapables de contribuer au chimérisme post-natal et/ou aux lignées germinales. On peut donc imaginer que la reprogrammation de certaines populations d'iPS est suffisamment «complète » pour qu'elles re-acquièrent un potentiel proche de celui de cellules épiblastiques très précoces, alors que d'autres s'arrêteraient en chemin, et seraient capables «seulement» de contribuer à un certain chimérisme post-natal, voire sans transmission germinale. On peut même induire une reprogrammation a minima au sein d'un tissu donné comme l'a très bien démontré $P$ Collombat pour le pancréas dans une dernière heure parue dans le dernier numéro de médecine/sciences [21]. Reste à identifier la signature génétique et épigénétique de ces nouveaux et différents états cellulaires chez la souris pour ensuite mieux classifier les iPS humaines dont nous ne connaissons pas vraiment le potentiel... Q'importe toute cette sémantique, diront certains..., c'est pourtant dans la précision de ces approches purement fondamentales aujourd'hui que se joue la réalité thérapeutique de demain redonnant à nos tissus l'ivresse de leurs jeunes années! Folle chimère... ? Pluripotency: defining standards 


\section{REMERCIEMENTS}

Merci à Michel Pucéat de sa relecture critique et de ses suggestions sur ce texte et à tous mes collègues dont les discussions ont nourri ce texte.

\section{CONFLIT D'INTÉRÊTS}

L'auteur déclare n'avoir aucun conflit d'intérêts concernant les données publiées dans cet article.

\section{RÉFÉRENCES}

1. Daley GQ, Lensch MW, Jaenisch R, et al. Broader implications of defining standards for the pluripotency of iPSCs. Cell Stem Cell 2009; $4: 200-1$.

2. Bonnevie L, Bel A, Sabbah L, et al. Is xenotransplantation of embryonic stem cells a realistic option? Transplantation $2007 ; 83: 333-5$.

3. Lensch MW, Schlaeger TM, Zon LI, Daley GQ. Teratoma formation assays with human embryonic stem cells: a rationale for one type of human-animal chimera. Cell Stem Cell $2007 ; 1: 253-8$

4. Bradley A, Evans M, Kaufman MH, Robertson $\varepsilon$. Formation of germ-line chimaeras from embryo- derived teratocarcinoma cell lines. Nature 1984 ; 309 : 255-6.

5. Thomas KR, Capecchi MR. Site-directed mutagenesis by gene targeting in mouse embryo-derived stem cells. Cell $1987 ; 51: 503-12$.

6. Keller $\mathrm{G}$. Embryonic stem cell differentiation: emergence of a new era in biology and medicine. Genes Dev 2005 ; 19 : 1129-55.

7. Nagy A, Gocza E, Diaz EM, et al. Embryonic stem cells alone are able to support fetal development in the mouse. Development $1990 ; 110: 815-21$.

8. Wanga Z, Jaenisch R. At most three ES cells contribute to the somatic lineages of chimeric mice and of mice produced by ES-tetraploid complementation. Dev Biol $2004 ; 275$ : 192-201.

9. Eggan K, Rode A, Jentsch I, et al. Male and female mice derived from the same embryonic stem cell clone by tetraploid embryo complementation. Nat Biotech $2002 ; 20: 455-9$

10. Tesar PJ, Chenoweth JG, Brook FA, et al. New cell lines from mouse epiblast share defining features with human embryonic stem cells. Nature 2007 ; 448: 196-9.

11. Takahashi K, Yamanaka S. Induction of pluripotent stem cells from mouse embryonic and adult fibroblast cultures by defined factors. Cell 2006; 126 : 663-76.

12. Wernig $M$, Meissner $A$, Foreman $R$, et al. In vitro reprogramming of fibroblasts into a pluripotent ES-cell-like state. Nature $2007 ; 448$ : 318-24.
13. Okita K, Ichisaka T, Yamanaka S. Generation of germline-competent induced pluripotent stem cells. Nature 2007 ; 448 : 313-7

14. Hochedlinger K, Plath K. Epigenetic reprogramming and induced pluripotency. Development 2009 ; $136: 509-23$.

15. Kang L, Wang J, Zhang $Y$, et al. iPS cells can support full-term development of tetraploid blastocystcomplemented embryos. Cell Stem Cell 2009; $5: 135-8$.

16. Zhao XY, Li W, Lv Z, et al. iPS cells produce viable mice through tetraploid complementation. Nature 2009; 461: 86-90.

17. Boland MJ, Hazen JL, Nazor KL, et al. Adult mice generated from induced pluripotent stem cells. Nature 2009 ; 461 : 91-94.

18. Monya Baker. Stem cells: Fast and furious. Nature $2008 ; 458: 962-5$

19. Silva J, Barrandon 0, Nichols J, et al. Promotion of reprogramming to ground state pluripotency by signal inhibition. PLoS Biol 2008 ; 6 : e253.

20. Ying $\mathrm{QL}$, Wray J, Nichols J, et al. The ground state of embryonic stem cell self-renewal. Nature 2008; 453: 519-23.

21. Collombat P, Mansouri A. Conversion de cellules $\alpha$ pancréatiques en cellules $\beta$. Med Sci (Paris) 2009; $25: 763-6$.

En dix ans. la pnẹmologie pédiatrique a connu un renouvellement exceptionnel, d’oà la néçessitẻ d'un volume consacté à cette spécialité

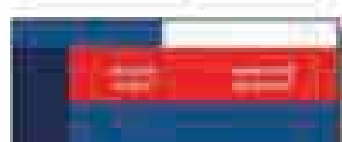

Pneumologie pediatrique

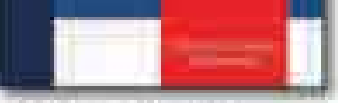

$23092009=455$ pager I86 ileutranians - 99 tableaur Wix pubstic risere

\section{PNEUMOLOGIE PÉDIATRIQUE} jacques DE BLIC, Christophe DELACOURT

La 1" partie de l'ouvrage est consacree atox - Outils diagnostiques -. On y trouvera en plarticulier

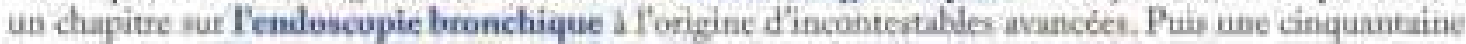
de chapitres sont consacres a la pathologie polmonaire chez f'enfant et Tadolescent. couvrunt tour il toar les pathologies : infecticuse, tumorale, immuno-ullergiqae, infitrative diffise, du sommeil, mais

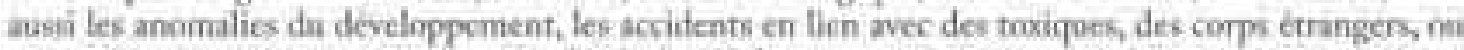
enconce les situatiota aigutes telles que T'embolie, la déttesse rospiratoirc. etc

Lu denière partic de louvrage eat plus particulièrement consactéc aux diverses prises en dairge en

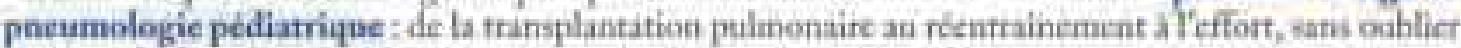
les cures hydrominérales et la kínésithénupic:-

\section{Au total, un recueil trés complet dont le caractère pluridisciplinaire et inmsversal sera d'un grand attrait pour les praticiens.}

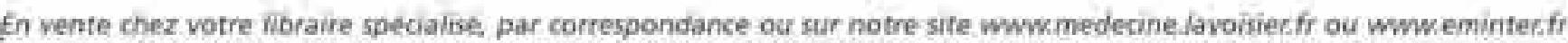

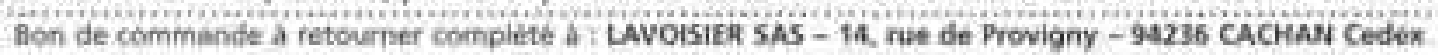

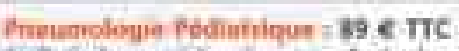

(*) $<$ de participation aux frais de port par exemplaine) soit $96 \&$
France mettopelstaine uniguemerit. Autres nocus conburtar? in joins mon reglement a la commanda z

Montant tatal ide

\section{e}

Cante hancairn $\mathrm{A}^{\prime}$

bate d'expination:

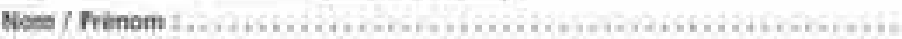

Fonction / specialite

Code postal :

vilie

Adresse:

tonit

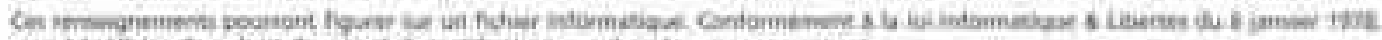

Date $n$ vignature obligetoine : 